\title{
RENDIMENTO E CARACTERIZAÇÃO DO CARVÃO VEGETAL DE GALHOS DE Mimosa caesalpiniifolia BENTH.
}

\author{
YIELD AND CHARACTERIZATION OF CHARCOAL FROM Mimosa caesalpiniifolia BENTH. \\ BRANCHES
}

\author{
Tarcila Rosa da Silva Lins ${ }^{1}$, Rafael Leite Braz², Carlos Geovani Cavalcanti de Souza Junior ${ }^{3}$, \\ Hudson Thiago Viana Correia ${ }^{4}$, Thiago Cardoso Silva ${ }^{5}$, Letícia Siqueira Walter ${ }^{6}$ \\ 1, 5,6Universidade Federal do Paraná, Curitiba, Paraná, Brasil - tarcila.lins@hotmail.com, \\ thiagocardoso.pe@gmail.com \& leticiasiqueira.walter@gmail.com \\ 2, 3, 4 Universidade Federal Rural de Pernambuco, Recife, Pernambuco, Brasil -rlbraz.ufrpe@gmail.com, \\ carlosgeovanisouza@gmail.com \&hudson_thiago@hotmail.com
}

\section{RESUMO}

A pesquisa teve como objetivo determinar o rendimento gravimétrico das carbonizações e caracterizar o carvão vegetal produzido a partir de galhos de Mimosa caesalpiniifolia Benth. (sabiá). Os galhos foram coletados no município de Camaragibe, localizado na Zona da Mata Norte do estado de Pernambuco, e fragmentados em cavacos, homogeneizados, e secos ao ar livre. Do material homogeneizado, foram obtidas dez amostras, que foram carbonizadas a fim de obter o rendimento gravimétrico e os teores de voláteis, cinzas, carbono fixo e poder calorífico do carvão vegetal. No processo de carbonização, os tratamentos utilizados corresponderam a duas taxas de aquecimento $\left(1,4^{\circ} \mathrm{C} / \mathrm{min}\right.$ e $\left.1,6^{\circ} \mathrm{C} / \mathrm{min}\right)$, com cinco repetições para cada, ambas com temperatura final de $460^{\circ} \mathrm{C}$. Estatisticamente, houve diferença para as variáveis materiais voláteis e rendimento gravimétrico, sendo mais altos no tratamento com maior taxa de aquecimento. Ao comparar os processos de carbonização, o tratamento com taxa de aquecimento de $1,6^{\circ} \mathrm{C}$ proporcionou melhor rendimento gravimétrico, porém os teores de carbono fixo e seus respectivos rendimentos em carvão vegetal, não diferiram para as taxas de aquecimento utilizadas. A partir das variáveis avaliadas, os resultados indicam que a madeira de $M$. caesalpiniifolia possui aptidão para uso energético e produção de carvão.

PALAVRAS-CHAVE: Taxa de aquecimento, Sansão-do-campo, Carbonização da madeira.

\section{ABSTRACT}

This study aimed was to determine the gravimetric yield of carbonisations and characterize the charcoal produced from M. caesalpiniifolia Benth. branches. The branches were collected in the municipality of Camaragibe, located in the Zona da Mata Norte of the state of Pernambuco, and were chipped, homogenized, and air dried. From the homogenized material, ten samples were obtained, which were carbonized in order to obtain the gravimetric yield and the content of volatile, ash, fixed carbon and calorific values of charcoal. In the carbonization process, the treatments used corresponded to two treatment heating rates $\left(1.4^{\circ} \mathrm{C} / \mathrm{min}\right.$ and $1.6^{\circ} \mathrm{C} / \mathrm{min}$ ), with five replicates for each, both with a final temperature of $460^{\circ} \mathrm{C}$. Statistically, there were differences for the variables volatile materials and gravimetric yield, being higher in the treatment with higher heating rate. The results indicated that the wood of $M$. caesalpiniifolia possesses aptitude for energy and coal production. When comparing the carbonization processes, the treatment with a heating rate of $1.6^{\circ} \mathrm{C}$ provided better gravimetric yield, but the fixed carbon contents and their respective charcoal yields did not differ for the heating rates used. From the evaluated variables, the results indicate that the wood of M. caesalpiniifolia possesses aptitude for energetic use and production of charcoal.

KEYWORDS: Heating rate, Sansão-do-campo, Wood carbonization. 


\section{INTRODUÇÃO}

$O$ estudo dos fatores que influenciam na qualidade do carvão é de grande importância, visto que é um dos principais pontos que determinam a sua aceitação entre os consumidores. O processo de produção do carvão vegetal, associado às características intrínsecas da espécie, é fundamental para determinar a qualidade do produto e como indicativo do uso final. Alguns fatores atrelados ao processo, como temperatura e taxa de aquecimento, exercem influência nas propriedades deste combustível, como o rendimento gravimétrico, teor de carbono fixo e de materiais residuais (TRUGILHO et al., 2015; SOUZA et al., 2016).

Utilizando diferentes metodologias de carbonização é possível perceber como as mudanças referentes a temperatura e velocidade de carbonização influenciam no rendimento gravimétrico e outras propriedades do carvão, pois com o aumento da taxa de carbonização ocorre a redução do rendimento do carvão e, consequentemente, o aumento do teor de materiais voláteis. Além disso, taxas de aquecimento maiores também proporcionam aumento no teor de cinzas (AZEVEDO et al., 2013).

A temperatura está relacionada ao teor de carbono fixo contido no carvão e no seu peso. Quando o processo é feito em altas temperaturas, ocorrerá maior fixação do carbono, porém terá redução no seu peso e tamanho, o que deixará o carvão mais frágil e poderá limitar sua aceitação no mercado (SOUZA et al., 2016).

Os materiais voláteis e cinzas correspondem ao material residual da decomposição térmica da madeira, sendo que os compostos minerais não contribuem para o processo. Por este motivo, não é desejável que a madeira para fins energéticos possua altos teores destes componentes, visto que reduzem o valor calórico do carvão. Além disso, o teor de materiais voláteis é inversamente proporcional ao teor de carbono fixo; espécies que possuem menores teores de materiais voláteis apresentam melhor rendimento, pois, queimam mais lentamente (REIS et al., 2012; LEITE et al., 2015).

As espécies de ocorrência no bioma Caatinga geralmente apresentam boas características para a produção de energia, uma delas é a Mimosa caesalpiniifolia Benth., popularmente conhecida como sabiá ou sansão do campo. É uma espécie pioneira e, por isso apresenta crescimento rápido, tem aplicação para diversas finalidades, permitindo o seu uso múltiplo. Sua casca possui alto teor de extrativos, principalmente taninos, e a madeira é classificada quanto a densidade como moderadamente densa a densa $\left(0,86\right.$ a 1,10 g. $\left.\mathrm{cm}^{-3}\right)$
(CARVALHO, 2007).

O sabiá normalmente é utilizado na função de quebravento ou cerca-viva devido à sua boa capacidade de rebrota. Quando são plantadas no entorno de uma propriedade, sua copa forma um "muro" que fornece proteção visual e evita a passagem de pessoas e animais, pois seus galhos contêm espinhos (RIBASKI et al., 2003).

A espécie estudada tem uso frequente como cerca viva ou quebra vento e uma das operações de manutenção das cercas vivas é a poda dos galhos, gerando resíduos que muitas vezes podem ter utilidade, mas são descartados. 0 sabiá apresenta boas qualidades como altos poder calorífico e resistência mecânica, o que a torna uma espécie de alto valor econômico para a região nordeste, apesar de suas características relacionadas à madeira não serem tão estudadas para tal potencial de uso (GONÇALVES et al., 2010).

A retirada periódica dos galhos é estimulada também por outras razões, como para a prevenção de incêndios, principalmente na estação seca. Segundo Brand (2017), durante este período de estiagem os galhos apresentam poucas folhas e menor teor de umidade, desta forma, podem contribuir para iniciar ou propagar incêndios, que ocorrem facilmente nesta época mais seca. A autora indica a retirada dos galhos nesta época também por causa facilidade de remoção desse material, que por estarem mais secos, apresentam menor resistência.

Apesar das espécies da Caatinga serem usadas de maneira intensiva para a produção de energia, não há muitas informações sobre o quantitativo de biomassa que é retirado do bioma para esta finalidade. Uma alternativa para a realização do uso sustentável destas espécies seria a implantação de programas de manejo, para conciliar a conservação com a geração de renda a partir da produção de carvão e lenha para a população inserida na caatinga (BRAND, 2017).

Neste contexto, o estudo foi realizado com o objetivo de verificar o potencial energético da madeira de Mimosa caesalpiniifolia Benth., avaliando a influência da taxa de aquecimento no rendimento gravimétrico em carvão vegetal, bem como realizar uma análise imediata do carvão vegetal.

\section{MATERIAL E MÉTODOS}

Foram coletados de maneira aleatória, galhos indivíduos de $M$. caesalpiniifolia, provenientes do sítio Bicho de Pé (754'24.3"S; 3459'57.0"W), situado no município de Camaragibe, localizado na Zona da Mata Norte do estado de Pernambuco. O clima da localidade 
segundo a classificação de Köppen, clima é tropical quente e úmido, com temperatura média de $26^{\circ} \mathrm{C}$ e com regime de chuvas de outono e inverno (SILVA, 2016).

Os galhos coletados foram transportados para o Laboratório de Tecnologia Florestal da Universidade Federal Rural de Pernambuco, apresentando um teor de umidade inicial de 51,6\%. Estes galhos foram fragmentados em cavacos com o auxílio de um facão, homogeneizados e secos ao ar livre até que atingissem a umidade de equilíbrio com o ambiente.

A densidade à granel dos galhos foi obtida de acordo com a norma NBR NM45 (ABNT, 2006), sendo realizadas cinco repetições, com resultado em $\mathrm{kg} \cdot \mathrm{m}^{-3}$.

As carbonizações foram realizadas em um forno do tipo mufla, com controle de temperatura, da marca MAGNU'S, modelo 0910. A carbonização foi conduzida num tempo total de 210 minutos a uma temperatura máxima de $460 \pm 10^{\circ} \mathrm{C}$ e duas taxas de aquecimento, $1,4^{\circ} \mathrm{C} / \mathrm{min} \mathrm{e}$ $1,6^{\circ} \mathrm{C} / \mathrm{min}$. A carga de cada carbonização era composta por uma amostra de aproximadamente $100 \mathrm{~g}$ de cavacos. Foram realizadas cinco repetições para cada taxa de aquecimento.

Cada amostra foi adicionada ao recipiente de aço inoxidável com dimensões $13 \mathrm{~cm} \times 15 \mathrm{~cm}$ x $8 \mathrm{~cm}$ (largura, comprimento e altura, respectivamente), com tampa e, introduzido na mufla previamente aquecida a $150^{\circ} \mathrm{C}$, mantendo-se esta temperatura por 30 minutos. Depois de transcorrido esse tempo, a temperatura foi elevada para $250^{\circ} \mathrm{C}$, permanecendo nesta temperatura por mais 30 minutos. Ao iniciar as reações exotérmicas, o tempo passou a ser medido a cada $20^{\circ} \mathrm{C}$, até atingir a temperatura máxima, conforme as taxas de aquecimento (Tabela 1).

Tabela 1. Temperaturas e taxas de aquecimento de cada tratamento.

\begin{tabular}{cccc}
\hline Tratamento & $\begin{array}{c}\text { Tempo de } \\
\text { duração }\end{array}$ & $\begin{array}{c}\text { Temperatura } \\
\text { final }\end{array}$ & $\begin{array}{c}\text { Taxa de } \\
\text { carbonização }\end{array}$ \\
\hline $\mathrm{T} 1$ & $210 \mathrm{~min}$ & $460 \pm 10^{\circ} \mathrm{C}$ & $1,4^{\circ} \mathrm{C} / \mathrm{min}$ \\
$\mathrm{T} 2$ & $195 \mathrm{~min}$ & $460 \pm 10^{\circ} \mathrm{C}$ & $1,6^{\circ} \mathrm{C} / \mathrm{min}$ \\
\hline
\end{tabular}

Ao finalizar a carbonização, a mufla foi desligada e iniciado o processo de resfriamento à temperatura ambiente por \pm 8 horas. Em seguida, foi determinado o rendimento gravimétrico em carvão vegetal (Equação 1).

$$
R G C=\left(\frac{\text { Pcarv }}{\text { Pmadeira }}\right) 100
$$

Em que: $\mathrm{RGC}=$ rendimento gravimétrico $\mathrm{da}$ carbonização (\%); Pcarv = peso do material carbonizado (g); e Pmadeira = peso da biomassa (g).
Posteriormente, o material carbonizado foi macerado e peneirado para a realização da análise imediata do carvão vegetal, conforme a NBR 8112 (ABNT, 1986). Foram determinados os teores de cinza, materiais voláteis e, por diferença, o teor de carbono fixo.

O poder calorífico superior (PSC) do carvão vegetal produzido foi obtido em bomba calorimétrica (calorímetro modelo IKA ${ }^{\circledR}$ WERKE C5003). A calibração do calorímetro foi realizada com base no aquecimento de ácido benzoico PA, resultando em $2959 \pm 30,4$ cal. $^{\circ} \mathrm{C}^{-1}$ (CV = 1,03\%), sendo este valor utilizado na base do cálculo do PCS, conforme a equação 2.

$$
Q=\frac{C x \Delta T}{m}
$$

Em que: $\mathrm{Q}=\mathrm{PCS}(\mathrm{cal} / \mathrm{g}) ; \mathrm{C}=2959 \pm 30,4 \mathrm{cal}^{\circ} \mathrm{C}^{-1} ; \Delta \mathrm{T}=$ variação de temperatura $\left({ }^{\circ} \mathrm{C}\right)$; e $\mathrm{m}=$ amostra de carvão $(\mathrm{g})$.

Os dados obtidos foram submetidos ao teste de normalidade de Shapiro-Wilk (W) e comparados por meio da Análise de Variância (ANOVA), realizou-se o Teste T para a comparação das médias.

\section{RESULTADOS E DISCUSSÃO}

A densidade à granel dos cavacos de galhos de sabiá obtida foi de $253,4 \mathrm{~kg} \cdot \mathrm{m}^{-3}$ ( $\mathrm{CV}=3,63 \%$ ), estando de acordo com o exposto por Brand (2010), que observou que a densidade de cavacos de madeira apresentam valores inferiores à densidade da madeira, num faixa entre $250 \mathrm{e}$ $350 \mathrm{~kg} \cdot \mathrm{m}^{-3}$.

Observou-se que houve diferença significativa entre os tratamentos para os rendimentos gravimétricos em carvão, ao comparar as diferentes marchas de carbonização, sendo possível observar uma uniformidade maior nos rendimentos em carbono fixo, que não variaram estatisticamente (Tabela 2).

Tabela 2. Valores referentes às variáveis rendimentos gravimétricos (\%) e de carbono fixo (\%).

\begin{tabular}{ccc}
\hline Tratamentos & \% RG & RCF \\
\hline T1 & 34,70 & 21,14 \\
T2 & 36,36 & 21,91 \\
Médias & 35,53 & 21,53 \\
CV & 1,86 & 2,72 \\
\hline
\end{tabular}

Os resultados encontrados para os rendimentos gravimétricos e em carbono fixo foram próximos aos valores expostos por Carvalho (2007), e o percentual médio de carbono fixo do presente estudo $(60,11 \%)$ foi 
inferior quando comparado ao trabalho citado (73\%). Ainda assim, estão satisfatórios, partindo do princípio que o material foi proveniente de estruturas jovens da árvore. A utilização dos galhos pode ser vista como uma forma alternativa de produzir energia, podendo promover maior sustentabilidade a esta prática.

O segundo tratamento apresentou maior percentual de materiais voláteis (Tabela 3 ), o que pode ter ocorrido devido ao aumento da taxa de carbonização para o mesmo. Os materiais voláteis sofrem influência de fatores como a temperatura de carbonização e taxa de aquecimento (OLIVEIRA, 1977), sendo assim, o acréscimo pode estar associado a esta mudança.

Tabela 3. Poder calorífico superior (PCS), teor de cinzas $(\% \mathrm{CZ})$, materiais voláteis $(\% \mathrm{MV})$, carbono fixo (\%CF).

\begin{tabular}{ccccc}
\hline Tratamentos & $\begin{array}{c}\text { PCS } \\
\text { (kcal/kg) }\end{array}$ & \%CZ & \%MV & \%CF \\
\hline T1 & 5944 & $5,51 \mathrm{a}$ & $33,60 \mathrm{a}$ & $60,89 \mathrm{a}$ \\
T2 & 5838 & $5,25 \mathrm{a}$ & $35,41 \mathrm{~b}$ & $59,34 \mathrm{a}$ \\
\hline Médias & 5891 & 5,38 & 34,50 & 60,11 \\
CV & - & 5.30 & 3.03 & 1.85 \\
\hline
\end{tabular}

A densidade à granel dos cavacos de galhos de sabiá obtida está de acordo com o exposto por Brand (2010), que observou que a densidade de cavacos de madeira apresenta valores inferiores à densidade da madeira, num faixa entre 250 e $350 \mathrm{~kg} \cdot \mathrm{m}^{-3}$.

Os valores de materiais voláteis e carbono fixos estão diretamente relacionados pois, o aumento de um resulta na diminuição do outro. O ideal é que o carvão vegetal apresente menor percentual de materiais voláteis, visto que correspondem aos resíduos volatilizados, e um maior quantitativo de carbono fixo que proporciona uma queima mais lenta e melhor rendimento do material nos fornos (STURION et al., 1988; REIS et al., 2012).

Apesar de reduzir o teor de carbono fixo, é preciso ter uma quantidade de materiais voláteis no carvão, pois proporcionam maior facilidade de ignição, estabilidade da chama e velocidade de combustão. Porém esta quantidade deve estar entre os $20 \%$ e $25 \%$, o alto teor de materiais voláteis faz com que seja produzida muita fumaça, o que não é desejável principalmente para o uso doméstico (SANTOS et al., 2016; SOUZA, et al. 2016).

$\mathrm{O}$ tratamento em que a maior taxa de aquecimento foi utilizada $\left(1,6^{\circ} \mathrm{C}\right)$, apresentou maiores percentuais de materiais voláteis e rendimento gravimétrico em comparação ao outro tratamento. Este comportamento é o inverso do que normalmente ocorre, segundo Azevedo et al. (2013), à medida que a taxa de aquecimento aumenta, essas variáveis tendem a reduzir.

Os teores de cinzas não diferiram entre si, porém ambos estão acima do que geralmente é esperado para espécies que são destinadas à produção de energia. $\mathrm{Na}$ literatura é indicado resultado de $0,45 \%$ de teor de cinzas para o sabiá (GONÇALVES et al., 2010).

O alto teor de cinzas não é desejável para espécies de interesse para produzir energia. Estes resíduos diminuem a eficiência do material combustível, pois necessitam de uma maior quantidade de oxigênio consumido para derreter as cinzas, além de apresentarem relação inversamente proporcional com o poder calorífico. Vale salientar que as cinzas estão presentes tanto na casca como no lenho e podem danificar os equipamentos por meio de corrosão (PEREIRA et al., 2016). Sugere-se que estes valores podem ter sido altos devido à presença das cascas na amostra, bem como resíduos de outra natureza (como poeiras) que estivessem aderidos a elas.

Os valores obtidos para o poder calorífico superior foram satisfatórios, com média de $5891 \mathrm{kcal} / \mathrm{kg}$, que é relativamente próximo aos valores médios encontrados por Brand (2017) que variam de 4583 a $4701 \mathrm{kcal} / \mathrm{kg}$ em estudo realizado com galhos de espécies da Caatinga, cujas espécies de maior abundância foram: Eugenia sp, Pisonia sp, Cenostigma gardnerianum Tul., Desmodium discolor Vogel, Luehea sp. Entretanto, vale ressaltar que a matéria prima utilizada para a análise do presente estudo foi proveniente do carvão vegetal, justificando o considerável aumento em relação aos valores anteriormente citados.

A utilização de taxas de aquecimento maiores reflete diretamente no rendimento do carvão de maneira negativa, visto que proporcionam aumento no teor de cinzas e materiais voláteis (AZEVEDO et al., 2013). No presente estudo, foi possível observar que a taxa de $1,6^{\circ} \mathrm{C}$ apresentou maior percentual de materiais voláteis e menor percentual de carbono fixo. Este comportamento é desejável pois o teor de voláteis está acima do esperado, e por consequência o teor de carbono fixo está inferior ao recomendado, como discutido anteriormente. Entretanto, a diferença entre taxas utilizadas foi baixa, resultando em valores com poucas diferenças consideráveis. Sugere-se, para estudos futuros, a utilização de uma amplitude maior de taxas de aquecimento para verificar outras possíveis influências na carbonização de galhos de sabiá para a produção de carvão vegetal.

Baseado nos resultados, percebe-se que os galhos do sabiá têm potencial de utilização para a produção de carvão vegetal devido às características apresentadas pela espécie. Desta forma, pode-se dar uma nova finalidade a 
um subproduto como os rejeitos de poda, de maneira a evitar o desperdício de um material que pode ser aproveitado como fonte de energia.

Apesar do uso intensivo de espécies de ocorrência no bioma Caatinga para a produção de energia, poucas informações ainda são conhecidas em relação às suas características. O conhecimento destas características é de suma importância, visto que a Caatinga é uma floresta seca com potencial de ser inserida no mercado energético, mas necessita de formas mais sustentáveis para a sua exploração (BRAND, 2017).

A obtenção de informações sobre o potencial de utilização de outras partes das árvores que não seja o fuste para a produção de energia, é de grande importância pois surge como uma alternativa para evitar o corte do mesmo para esta finalidade. Portanto, o uso dos galhos para produzir carvão vegetal poderia ser proposto como forma de uso sustentável destas espécies, para tentar contornar, por exemplo, o uso intensivo que ocorre na caatinga (local de onde essa espécie é nativa) que vem acontecendo no decorrer dos anos e resulta em impactos negativos para a vegetação.

\section{CONCLUSÕES}

O aumento da taxa de aquecimento exerce influência no rendimento gravimétrico e no teor de materiais voláteis. O tratamento com a taxa de aquecimento de $1,4^{\circ} \mathrm{C}$ apresentou melhores resultados de poder calorífico e carbono fixo, características importantes para a produção de energia.

A utilização dos galhos de sabiá pode ser indicada para a produção de energia através de sua conversão em carvão vegetal, visto que, de modo geral, as variáveis avaliadas apresentaram valores satisfatórios para esta finalidade. 0 uso de outra parte da árvore que não seja o fuste principal pode servir como uma alternativa que promova o uso sustentável desta espécie.

Sugere-se a retirada das cascas antes de realizar o procedimento para prevenir possíveis problemas com altos teores de resíduos minerais e evitar danos aos equipamentos utilizados.

\section{REFERÊNCIAS}

ABNT - ASSOCIAÇÃO BRASILEIRA DE NORMAS TÉCNICAS. NBR 8112: Carvão Vegetal - Análise Imediata. Rio de Janeiro: ABNT, 1986.

ABNT - ASSOCIAÇÃO BRASILEIRA DE NORMAS TÉCNICAS. NBR NM45: Agregados - Determinação da massa unitária e do volume de vazios. Rio de Janeiro: ABNT, 2006.
AZEVEDO, C.H.S. et al. Influência da temperatura final de carbonização e da taxa de aquecimento no rendimento gravimétrico e teor de cinzas do carvão de Eucalyptus urophylla $\mathrm{x}$ Eucalyptus grandis. Enciclopédia Biosfera, v.9, n.16, p.1279.1289, 2013.

BRAND, M.A. Potencial de uso da biomassa florestal da caatinga, sob manejo sustentável, para geração de energia. Ciência Florestal, v.27, n.1, p.117-127, 2017.

BRITO, J.O.; BARRICHELO, L.E.G. Aspectos técnicos da utilização da madeira e carvão vegetal como combustíveis. 2o Seminário de Abastecimento Energético Industrial com Recursos Florestais, p.101-137, 1982.

CARVALHO, P.E.R. Sabiá - Mimosa caesalpiniifolia. Circular técnica, 135. Colombo: Embrapa Florestas, 2007.

GONÇALVES, C.A. et al. Caracterização físico-química da madeira de sabiá (Mimosa caesalpiniaefolia Benth.). Revista Caatinga, v.23, n.1, p.54-62, 2010.

LEITE, E.R.S. et al. Qualidade do carvão vegetal produzido a partir de madeira do cafeeiro, para uso bioenergético. Coffee Science, v.10, n.2, p.251-261, 2015.

OLIVEIRA, J.V. Análise econômica do carvão vegetal. 10 Seminário sobre Carvão Vegetal, 1977.

PEREIRA, M.P.C.F. et al. Torrefação de cavacos de eucalipto para fins energéticos. Pesquisa florestal brasileira, v.36, n.87, p.269275, 2016.

REIS, A.A. et al. efeito de local e espaçamento na qualidade do carvão vegetal de um clone de Eucalyptus urophylla S. T. Blake. Floresta e Ambiente, v.19, n.4, p.497-505, 2012.

RIBASKI, J. et al. Sabiá (Mimosa caesalpiniaefolia): árvore de múltiplo uso no Brasil. Colombo: Embrapa Florestas, 2003.

SANTOS, R.C. et al. Influência das propriedades químicas e da relação siringil/guaiacil da madeira de eucalipto na produção de carvão vegetal. Ciência Florestal, v.26, n.2, p.657-669, 2016.

SILVA, A.B. Da problemática urbana à emergência da vida associada à natureza: motivações para viver em Aldeia (Camaragibe-PE). Tamoios, v.12, n.2, p.112-123, 2016.

SOUZA, N.D. et al. Estudo de caso de uma planta de carbonização: Avaliação de características e qualidade do carvão vegetal visando uso siderúrgico. Floresta e Ambiente, v.23, n.2, p.270$277,2016$.

STURION, J.A. et al. Qualidade da madeira de Eucalyptus vimanalis para fins energéticos em função do espaçamento e idade de corte. Boletim de Pesquisa Florestal, n.16, p.55-59, 1988.

TRUGILHO, P.F. et al. Efeito da idade e material genético no rendimento e qualidade do carvão vegetal de eucalipto. Ciência da Madeira, v.6, n.3, p.202-2016, 2015.

Recebido em 12-06-2019 Aceito em 29-07-2019 